\title{
Improving Online Accessibility for Individuals with Photosensitive Epilepsy
}

\author{
LAURA SOUTH, Northeastern University \\ Content with flashes, bright colors, and repeated patterns can cause seizures and migraines when viewed by people with photosensitivity. \\ Exposure to seizure-inducing content is a serious risk in online environments, as evidenced by documented instances of people with \\ photosensitivity being exposed to seizure-inducing material while playing video games or using social media. My thesis focuses on \\ improving online safety and accessibility for people with photosensitivity by measuring the prevalence of seizure-inducing content \\ online, developing new tools for detecting seizure-inducing content, and constructing a broad framework for protecting against \\ seizure-inducing content at the level of content creators, platforms, and content consumers. Through this work, I hope to help build a \\ better understanding of the current state of photosensitive risk online and contribute new solutions for mitigating seizure-inducing \\ content with minimal adverse effects on the browsing experience for users with photosensitivity.
}

Additional Key Words and Phrases: accessibility, photosensitive epilepsy

\section{INTRODUCTION}

Individuals with photosensitivity experience seizures, migraines, and other symptoms when exposed to specific forms of light and pattern stimuli. Seizures can be triggered by a wide variety of phenomena encountered in daily life, such as fireworks [12], concert lighting [18], and wind turbines [13]. Encountering seizure-inducing content is a particular risk when interacting with technology. People with photosensitivity have reported seizures triggered while playing video games [19], engaging with social media [14], and even taking selfies with a mobile phone [5].

Despite the obvious risks of encountering seizure-inducing content when using technology, much remains unclear about the accessibility of digital spaces for individuals with photosensitivity. At the most basic level, we have little sense of the prevalence of seizure-inducing content across various online contexts - i.e., how bad is the problem? For example, isolated case studies of flashing GIFs being weaponized to cause seizures online have been reported since at least 2008, but no large-scale studies of seizure-inducing content in GIFs were completed until 2021 (Section 3.1). Very little work has been done to understand adherence to accessibility guidelines related to photosensitivity (e.g., Guideline 2.1 in WCAG 2.0 [6]), making it difficult for nonprofit groups such as the Epilepsy Foundation ${ }^{1}$ and the Epilepsy Society ${ }^{2}$ to effectively advocate for better protections for people with photosensitivity. The lack of studies investigating online accessibility as it relates to photosensitivity can in part be attributed to the limitations of currently available software tools for identifying photosensitive risk factors in online content. The leading free photosensitive risk detection system (Photosensitive Epilepsy Analysis Tool ${ }^{3}$, or PEAT) was built to analyze screen recordings and longer-form videos and is not suitable for accurately testing short, looping GIFs. PEAT is intended to be used by content creators to test for

\footnotetext{
${ }^{1}$ https://www.epilepsy.com/

${ }^{2}$ https://epilepsysociety.org.uk/

${ }^{3}$ https://trace.umd.edu/peat/
}

Author's address: Laura South, Northeastern University, south.l@northeastern.edu.

Permission to make digital or hard copies of all or part of this work for personal or classroom use is granted without fee provided that copies are not made or distributed for profit or commercial advantage and that copies bear this notice and the full citation on the first page. Copyrights for components of this work owned by others than ACM must be honored. Abstracting with credit is permitted. To copy otherwise, or republish, to post on servers or to redistribute to lists, requires prior specific permission and/or a fee. Request permissions from permissions@acm.org.

(c) 2021 Association for Computing Machinery.

Manuscript submitted to ACM 
photosensitive risk before releasing videos online and does not allow for batch processing, making it challenging for researchers to conduct large-scale studies of seizure-inducing content.

In my thesis, I aim to improve online safety and accessibility for people with photosensitivity through three different approaches. First, I will continue building tools for automatically detecting seizure-inducing content that are available and useful for both individuals with photosensitivity and accessibility researchers. Second, I will continue measuring the prevalence of seizure-inducing content through large-scale studies of online artifacts and testing adherence to established accessibility guidelines for photosensitivity. Third, I will continue to refine and clarify my framework for eliminating exposure to seizure-inducing content by relying on consumer-, platform-, and creator-driven layers of protection. In the following sections I will describe my preliminary work on seizure-inducing GIFs (Section 3.1) and seizure-inducing data visualizations (Section 3.2), as well as my proposed work on seizure-inducing content in virtual reality and the potential for individualized risk detection systems. All four projects use these three approaches to provide a deeper understanding of the current state of accessibility for people with photosensitivity and to illuminate a path forward where the many online artifacts discussed above have less potential for harm.

\section{RELATED WORK}

The preliminary and proposed work described in this paper is informed and influenced by prior work in two other areas of research: online accessibility for people with disabilities other than photosensitivity and automated detection of photosensitive risk factors.

\subsection{Accessibility}

My work on measuring adherence to WCAG guidelines for photosensitivity in GIFs and interactive data visualizations (Section 3.1 and 3.2) is influenced in large part on similar studies measuring website accessibility for users with vision impairments (e.g., $[1,15,17])$. Gleason et al.'s study of accessibility of GIFs for users with vision impairments is closely related to the work on seizure-inducing GIFs described in Section 3.1. There are several key browser extensions that provide consumer-driven improvements to web accessibility which were formative in developing PhotosensitivityPal, such as Twitter A11y [10], Caption Crawler [11], and Firefixia [8].

\subsection{Detection of photosensitive risk factors}

My research into accessibility for people with photosensitivity is built on a large and comprehensive body of work aimed at defining photosensitive risk factors. Fisher et al. summarize the results of multiple EEG studies that found the specific characteristics that are commonly found in flashing sequences that cause seizures (e.g., rate of flashes, area covered by flashes, luminance difference between flashes) [9]. Wilkins et al. provide a similarly thorough explanation of the risk factors that can cause a repeated pattern to be hazardous [22]. My work is also informed by a handful of implementations of photosensitive risk detection systems that use machine learning (e.g., [4]) or rule-based (e.g., [3]) approaches for risk detection.

\section{COMPLETED WORK}

\subsection{Seizure-inducing GIFs}

GIFs are short, looping videos that are ubiquitous on many social media platforms. Despite their reputation as lighthearted methods of communicating tone in online communities [16], GIFs are frequently used to orchestrate malicious

Manuscript submitted to ACM 


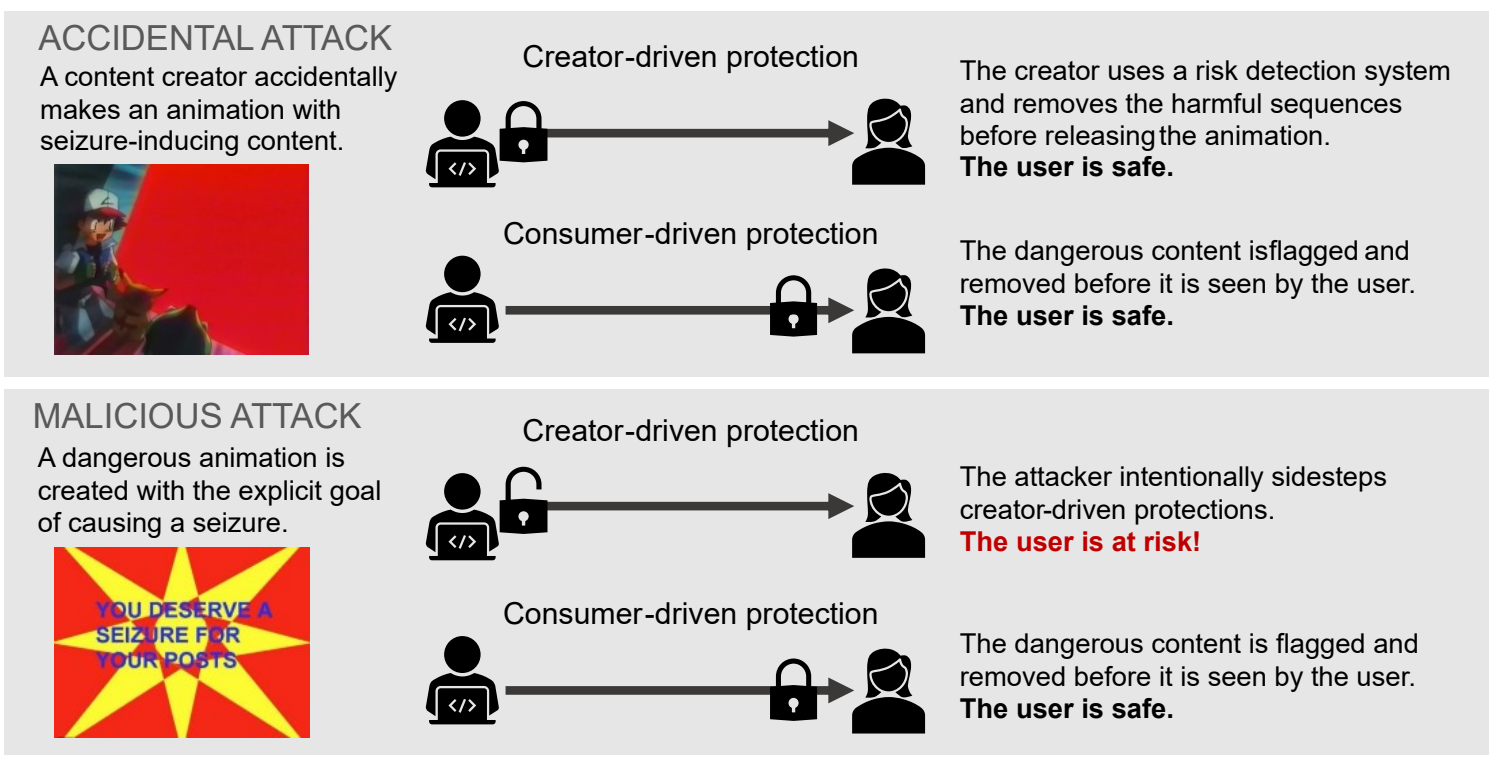

Fig. 1. Creator-driven systems rely on content creators to actively check their work for seizure-inducing content before releasing it online, leaving those with photosensitivity vulnerable when creators avoid such protections out of ignorance or malice. Consumerdriven systems protect users in both accidental and malicious attack scenarios.

attacks against people with photosensitivity online. In recent years, attacks against journalist [14], the Epilepsy Society [21], and the Epilepsy Foundation [2] have all been conducted using GIFs with strobing or flashing sequences. Because of the prevalence of animated GIFs in these malicious attacks, I chose to focus my first study into online accessibility for people with photosensitivity within the context of animated GIFs encountered on social media.

We conducted a series of interviews with people with photosensitivity that use social media regularly and found that most participants felt uncomfortable and at risk of accidentally encountering seizure-inducing content while using the internet. They felt that there were few options available to them to help avoid seizure-inducing content except for low-tech solutions such as asking friends to check for dangerous content in videos for them or relying on content creators to include warnings about strobes or flashes. To address this gap, we built PhotosensitivityPal, a browser extension that automatically scans for seizure-inducing content in all GIFs the user encounters. We also introduced a framework for categorizing photosensitive risk detection systems as consumer-driven, platform-driven, or creator-driven. PhotosensitivityPal was a prototype example of a consumer-driven system because it allowed the end user to have control over what content was blocked, rather than relying on platforms or content creators to decide if content is photosensitive safe or not. Figure 1 contrasts creator- and consumer-driven protection systems for two examples of seizure-inducing GIFs found online. PhotosensitivityPal served a dual purpose by helping users with photosensitivity browse the internet more safely while also providing a way for researchers to test GIFs for photosensitive risk in bulk far more efficiently. This allowed us to conduct the first large-scale study of photosensitive risk factors in GIFs posted to social media. We found that approximately $8 \%$ of 2000 GIFs collected for the study had the potential to cause seizures when viewed by someone with photosensitivity, establishing the severity of the problems faced by users with photosensitivity on social media. The development of PhotosensitivityPal and its subsequent use in the our quantitative study of seizure-inducing GIFs on social media were formative in shaping the rest of my thesis; 
once it became clear that we could use this approach to understand and improve accessibility of GIFs on social media, I knew that we could expand the approach to examine other contexts beyond social media.

\subsection{Seizure-inducing data visualizations}

My first project in accessibility research focused on identifying and mitigating seizure-inducing content in GIFs posted to social media (Section 3.1), but as I continued to explore the research space I realized that there was a need to better understand photosensitive risk in other online contexts beyond social media. I chose to investigate the risks people with photosensitivity face when navigating interactive online data visualizations, a content format that is more common than GIFs in professional and mission-critical contexts. The potential for seizure-inducing data visualizations was established in 2005 by Conti et al., although they refrained from performing a comprehensive study on the specific ways in which a data visualiation could cause seizures [7]. Interactive visualizations are non-deterministic, making them more challenging to test for photosensitive risk than a deterministic GIF that will play exactly the same sequence regardless of context. An interactive visualization could be completely innocuous and safe when used by a careful user who interacts with the system slowly and without sudden movements, but the same visualization might produce seizure-inducing sequences if the user interacts with sharp, sudden movements.

We collected screen recordings of interactions with over 1000 interactive visualizations and used PEAT to test for photosensitive risk factors. Approximately $8 \%$ of the visualizations included in our study were capable of producing seizure-inducing sequences through interaction. We categorized the potentially dangerous visualizations into eight hazard categories based on the interaction techniques used to produce the dangerous sequences (i.e., click, zoom, hover) and contributed mitigation techniques that visualization creators can use to avoid inadvertently creating webpages with seizure-inducing sequences in the future. As a result of this project, we have a better understanding of how webpages with interactive data visualizations can be made safer for users with photosensitivity.

\section{PROPOSED WORK}

\subsection{Seizure-inducing content in virtual reality}

Virtual reality (VR) is growing in popularity as a way to play video games, communicate with others, watch immersive videos, and more. VR platforms pose a particular challenge for people with photosensitivity because VR headsets occupy almost the entire field of view. Research has shown that photosensitive risk is strongly correlated with the area of retina stimulated by the strobing or flashing content [9], so it is likely that content that is safe when viewed on a laptop or phone could become seizure-inducing when viewed in an immersive VR context that stimulates a larger percentage of the retina. Interview participants with photosensitivity have described a low-tech solution they use for mitigating seizure-inducing content on a phone or laptop, which is to quickly remove the offending device by shutting the laptop or throwing the phone away from [20]. Getting away from the dangerous content is more involved when the user is wearing a VR headset, making it even more important to establish protective systems within VR software to automatically detect and block seizure-inducing content.

I plan to conduct a study investigating seizure-inducing content in the context of VR, including estimate of the prevalence of dangerous content in various types of VR media (i.e., video games, immersive videos, social platforms). This project will also incorporate the development and testing of a new software for automatically detecting seizureinducing content in VR, similar to the browser extension for testing GIFs (PhotosensitivityPal) that was described in Section 3.1.

Manuscript submitted to ACM 


\subsection{Individualized photosensitive risk thresholds}

Not all people with photosensitivity will experience the same sensitivity to the three primary risk factors identified in EEG trials as common triggers (flashes, repeated patterns, and transitions to and from saturated red). While some people might be extremely sensitive to content with repeated striped patterns, others will not be affected at all. Additionally, Fisher et al. observed in EEG trials that the precise thresholds at which a sequence becomes problematic differs at an individual level [9]. Fisher et al. aggregated individual results to produce general guidelines that would prevent seizures for most people with photosensitivity when applied unilaterally to content that would be viewed by many people, such as television programs or films. However, a major advantage of a consumer-driven system such as PhotosensitivityPal is that it has the potential to be customized for the specific person using the browser extension, rather than implementing generalized rules that may or may not be appropriate for the sensitivities of the current user.

I plan to implement an updated version of PhotosensitivityPal that can be customized by the user to detect and block content that exceeds thresholds specified by the user. Based on the interviews I conducted for my study of seizure-inducing GIFs (Section 3.1), people with photosensitivity are often aware of what content is most triggering for them. By allowing users with photosensitivity to have more control over what content is automatically blocked, we can increase their autonomy over their browsing experience while still providing an extra layer of protection against accidental or malicious exposure to dangerous content. I will conduct a mixed methods evaluation of my personalized risk detection system to determine if such an individualized approach is beneficial to people with photosensitivity when interacting online.

\section{QUESTIONS FOR THE PANEL}

I welcome all feedback the panel may have regarding the work I have proposed, in addition to responses to several specific questions. First, does the panel have thoughts on the correct balance of developing prototype systems for improving accessibility and conducting studies to measure the current state of accessibility in various online contexts? Should I focus on one type of research over the other? Second, is the scope of my proposed projects suitable for the remaining one to two years of my $\mathrm{PhD}$ ? Finally, I would love to know if the panel has advice for connecting with accessibility advocacy groups, such as the Epilepsy Foundation, to make sure that my research is benefiting the epilepsy community as much as possible, rather than only influencing researchers within the HCI community.

\section{REFERENCES}

[1] Amaia Aizpurua, Simon Harper, and Markel Vigo. 2016. Exploring the relationship between web accessibility and user experience. International Journal of Human-Computer Studies 91 (2016), 13-23.

[2] Jackie Aker. 2019. Epilepsy Foundation Files Criminal Complaint and Requests Investigation in Response to Attacks on Twitter Feed. Epilepsy Foundation (16 Dec. 2019). https://www.epilepsy.com/release/2019/12/epilepsy-foundation-files-criminal-complaint-and-requests-investigationresponse

[3] Mohammad A Alzubaidi, Mwaffaq Otoom, and Abdel-Karim Al-Tamimi. 2016. Parallel Scheme for Real-Time Detection of Photosensitive Seizures. Computers in Biology and Medicine 70 (2016), 139-147.

[4] Andrei Barbu, Dalitso Banda, and Boris Katz. 2019. Deep Video-to-Video Transformations for Accessibility with an Application to Photosensitivity. Pattern Recognition Letters (2019).

[5] PM Brna and KG Gordon. 2017. "Selfie-epilepsy": A novel photosensitivity. Seizure 47 (2017), 5-8.

[6] Ben Caldwell, Michael Cooper, Loretta Guarino Reid, and Gregg Vanderheiden. 2008. Web Content Accessibility Guidelines (WCAG) 2.0. WWW Consortium (W3C) (2008).

[7] Gregory Conti, Mustaque Ahamad, and John Stasko. 2005. Attacking information visualization system usability: overloading and deceiving the human. In Proceedings of the 2005 Symposium on Usable Privacy and Security. 89-100.

[8] Vagner Figueredo de Santana, Rosimeire de Oliveira, Leonelo Dell Anhol Almeida, and Marcia Ito. 2013. Firefixia: An accessibility web browser customization toolbar for people with dyslexia. In Proceedings of the 10th International Cross-Disciplinary Conference on Web Accessibility. 1-4. 
[9] Robert S Fisher, Graham Harding, Giuseppe Erba, Gregory L Barkley, and Arnold Wilkins. 2005. Photic- and Pattern-induced Seizures: a Review for the Epilepsy Foundation of America Working Group. Epilepsia 46, 9 (2005), 1426-1441.

[10] Cole Gleason, Amy Pavel, Emma McCamey, Christina Low, Patrick Carrington, Kris M Kitani, and Jeffrey P Bigham. 2020. Twitter A11y: A Browser Extension to Make Twitter Images Accessible. In Proceedings of the 2020 CHI Conference on Human Factors in Computing Systems. 1-12.

[11] Darren Guinness, Edward Cutrell, and Meredith Ringel Morris. 2018. Caption Crawler: Enabling reusable alternative text descriptions using reverse image search. In Proceedings of the 2018 CHI Conference on Human Factors in Computing Systems. 1-11.

[12] Gürkan Gürbüz and Özge Berfu Gürbüz. 2021. A rare trigger for photosensitive seizure: fireworks. Acta Neurologica Belgica (2021), 1-4.

[13] Graham Harding, Pamela Harding, and Arnold Wilkins. 2008. Wind turbines, flicker, and photosensitive epilepsy: Characterizing the flashing that may precipitate seizures and optimizing guidelines to prevent them. Epilepsia 49, 6 (2008), 1095-1098.

[14] Cecilia Kang. 2017. A Tweet to Kurt Eichenwald, a Strobe, and a Seizure. Now, an Arrest. The New York Times (2017)

[15] Jennifer Mankoff, Holly Fait, and Tu Tran. 2005. Is your web page accessible? A comparative study of methods for assessing web page accessibility for the blind. In Proceedings of the SIGCHI Conference on Human Factors in Computing Systems. 41-50.

[16] Kate M Miltner and Tim Highfield. 2017. Never Gonna GIF You Up: Analyzing the Cultural Significance of the Animated GIF. Social Media+ Society 3, 3 (2017), 2056305117725223.

[17] Christopher Power, André Freire, Helen Petrie, and David Swallow. 2012. Guidelines are only half of the story: accessibility problems encountered by blind users on the web. In Proceedings of the 2012 CHI Conference on Human Factors in Computing Systems. 433-442.

[18] Newel Salet, Marieke Visser, Cornelis Stam, and Yvo M Smulders. 2019. Stroboscopic light effects during electronic dance music festivals and photosensitive epilepsy: a cohort study and case report. BMJ open 9, 6 (2019), e023442.

[19] Mohammadali M Shoja, R Shane Tubbs, Armin Malekian, Amir H Jafari Rouhi, Mohammad Barzgar, and W Jerry Oakes. 2007. Video game epilepsy in the twentieth century: a review. Child's Nervous System 23, 3 (2007), 265-267.

[20] Laura South, David Saffo, and Michelle A Borkin. 2021. Detecting and Defending Against Seizure-Inducing GIFs in Social Media. In Proceedings of the 2021 CHI Conference on Human Factors in Computing Systems. 1-17.

[21] Nicola Swanborough. 2020. Epilepsy Society Sees Worst Ever Bullying Attack On Twitter. Epilepsy Society (16 May 2020). https:/www. epilepsysociety.org.uk/news/Epilepsy-Society-sees-worst-ever-attack-Twitter-16-05-2020

[22] Arnold Wilkins, John Emmett, and Graham Harding. 2005. Characterizing the patterned images that precipitate seizures and optimizing guidelines to prevent them. Epilepsia 46, 8 (2005), 1212-1218. 